\title{
Arthroscopic Debridement of Elbow Osteoarthritis Using CT-Based Computer-Aided Navigation Systems Is Accurate
}

\author{
Ryoya Shiode, M.D., Kunihiro Oka, M.D., Atsuo Shigi, M.D., Satoshi Miyamura, M.D., \\ Hiroyuki Tanaka, M.D., Tatsuo Mae, M.D., and Tsuyoshi Murase, M.D.
}

\begin{abstract}
Purpose: To evaluate whether the bony impingement lesion in elbow osteoarthritis can be removed accurately, as planned during arthroscopy, by using the computer-aided navigation system and performing mock surgery using 3-dimensional (3D)printed bone models for clinical applications. Methods: We performed mock surgery using 3D-printed plaster bone models of the humerus of 15 actual patients with elbow osteoarthritis. Two types of experiments were conducted to evaluate the surgical accuracy. Three surgeons performed the mock surgery, each with 15 bone models (total, 45 trials). Surgical accuracy was based on the mean of 45 trials. The differences in surgical accuracy among the 3 surgeons were also evaluated (mean 15 trials). The same surgeon performed 30 trials, and the difference in surgical accuracy between the first and the second halves was also evaluated (mean 15 trials). Results: The spatial error in the entire elbow joint was $1.13 \mathrm{~mm}$. In terms of resection volume, a mean of $8 \%$ more volume was resected than was planned, and $85 \%$ of the planned area was resected. In our experiments, the surgical accuracy was significantly lower in the anterior than in the posterior joint. Intrarater reliability was intraclass correlation (ICC) $)_{2,1} 0.81$ and inter-rater reliability was ICC $_{1,1} 0.87$. Conclusions: Surgery using computer-aided navigation systems for arthroscopic debridement of the elbow provided accuracy comparable to that in other joints. Clinical Relevance: Arthroscopic debridement of elbow osteoarthritis requires advanced surgical skills because accurate identification of the bony impingement legion is difficult during surgery. Surgery using computer-aided navigation systems for arthroscopic debridement of the elbow will provide real-time tracking of both the surgical instruments and bony impingement lesions as well as solve the technical difficulties of arthroscopic surgery of the elbow joint.
\end{abstract}

From the Department of Orthopaedic Surgery, Graduate School of Medicine, Osaka University, Suita (R.S., K.O., S.M., H.T., T.M., T.M.) and Yukioka Hospital Hand Center, Osaka-shi (A.S.), Osaka, Japan.

The authors report the following potential conflicts of interest or sources of funding: K.O. and T.M. received funding in support of this research from the Japan Society for the Promotion of Science. The other authors have no disclosures to report. This work was supported by JSPS KAKENHI Grant \#JP 15K10442 and JP 15H04957. Full ICMJE author disclosure forms are available for this article online, as supplementary material.

This study was approved by the institutional review board at our hospital (approval \#15426), which waived the need for informed consent from each participant.

Received February 11, 2021; accepted July 25, 2021.

Address correspondence to Kunihiro Oka, M.D., Department of Orthopaedic Surgery, Graduate School of Medicine, Osaka University, 2-2, Yamadaoka, Suita, Osaka 565-0871, Japan.E-mail:oka-kunihiro@umin.ac.jp

(C) 2021 THE AUTHORS. Published by Elsevier Inc. on behalf of the Arthroscopy Association of North America. This is an open access article under the CC BY-NC-ND license (http://creativecommons.org/licenses/by-nc-nd/4.0/).

2666-061X/21147

https://doi.org/10.1016/j.asmr.2021.07.025
$\mathbf{P}$ rimary elbow osteoarthritis is a relatively rare condition that occurs mainly in middle-aged men who experienced frequent loading of the upper extremity, such as those performing manual labor and athletes. ${ }^{1,2}$ Patients with elbow osteoarthritis usually have osteophytes and capsular contractures, resulting in pain at the endpoints of the range of motion with some loss of terminal extension and flexion. ${ }^{2-6}$ One treatment to recover elbow function is debridement arthroplasty, including removal of osteophytes, synovectomy and release of the contracted capsule via open $^{2,6}$ or arthroscopic ${ }^{1,7-10}$ surgery. In particular, less invasive arthroscopic surgery can reduce surgical complications and is expected to contribute to early functional recovery of the elbow. ${ }^{11}$ However, arthroscopic elbow surgery requires experienced surgical skills compared to open surgery because of the limited arthroscopic view of the working space and the complicated 3-dimensional (3D) shape of the elbow joint. ${ }^{12}$ Therefore, accurate identification of the bony impingement lesion is difficult during surgery. 
The focus of the present study was the evaluation of computer-aided surgery based on virtual surgical planning for elbow arthroscopy to assist the surgeon during surgery. A preoperative simulated program was reported to identify the bony impingement lesion to be removed using 3D models of the elbow in multiple positions created from computed tomography (CT) data. ${ }^{13}$ In addition, computer-aided navigation systems for use during arthroscopic elbow surgery have recently been validated and have reported registration accuracy of 0.96 and $0.85 \mathrm{~mm}$ for the humerus and ulna, respectively. ${ }^{14}$ By combining preoperative simulation and the computer-aided navigation systems, surgery may be performed according to the preoperative simulation with high efficiency and minimal invasiveness.

The purpose of this study was to evaluate whether a bony impingement lesion in elbow osteoarthritis can be removed accurately, as planned during arthroscopy, using the computer-aided navigation system by performing mock surgery using 3D-printed bone models for clinical applications. The hypotheses for this study were as follows: surgery using computer-aided navigation system for arthroscopic debridement of the elbow is sufficiently accurate for clinical applications; and surgical accuracy does not significantly differ between the anterior and posterior areas and among surgeons.

\section{Methods}

This is a validation study of the surgical accuracy of arthroscopic debridement of the elbow using a CTbased navigation system and preoperative 3D surgical simulation. All procedures were performed in accordance with the ethical standards of the responsible committees on human experimentation (institutional and national) and with the Declaration of Helsinki of 1975, as revised in 2000.

\section{Specimens and Preoperative Simulation}

CT data were extracted from all patients with elbow osteoarthritis visiting our institution between September 2014 and October 2018 who underwent CT scanning at 3 elbow positions (maximum extension, $90^{\circ}$ flexion and maximum flexion) to analyze the impingement lesion for surgery in accordance with the preoperative simulation program in the previous report. ${ }^{13} \mathrm{CT}$ images of the whole humerus, radius and ulna were acquired using a helical CT scanner (LightSpeed Ultra 16; General Electric, Waukesha, WI, USA) with a low-dose radiation protocol ${ }^{15}(120-\mathrm{kV}$ tube voltage, 30-mA current, $1.25 \mathrm{~mm}$-thick slices, and 0.48 $\mathrm{mm}$ pixel size). During scanning, the upper limb was elevated over the head with the subject in a prone position, and the forearm was in supination. CT data of the 3 elbow positions were sent to a workstation in
Digital Imaging and Communications in Medicine (DICOM) format. The 3D computer bone models of the humerus, radius and ulna were reconstructed in Standard Triangulation Language (STL) format from the CT data by using dedicated threshold-based segmentation software (BoneViewer; Orthree, Osaka, Japan) with a 200 Hounsfield unit threshold. ${ }^{16,17}$

To identify the location of the bony impingement lesion, the normal elbow range of motion (flexion range of motion $0^{\circ}-140^{\circ}$ ) was simulated by $3 \mathrm{D}$ models of the 3 elbow positions by using a commercially available software program (Bone Simulator; Orthree). Surface-based registration was performed for the humerus and 3D models of the 3 positions were superimposed on the basis of the humerus. The rotational axis of the ulna was calculated relative to the humerus between each position. The axis between maximum extension and $90^{\circ}$ flexion was defined as the extension axis and that between $90^{\circ}$ flexion and maximum flexion as the flexion axis (Fig 1). The bone position was simulated at $0^{\circ}$ and $140^{\circ}$ of the elbow by rotating the ulna and radius from maximum extension around the extension axis and from maximum flexion around the flexion axis, and the area where the humerus, radius and ulna overlapped was defined as the bony impingement lesion (Fig 2).

Based on the CT data, 3D-printed bone models (ZPrinter 450; Z, Burlington, MA, USA) with plaster $\left(\mathrm{zp}^{\mathrm{pR}} 150 ; \mathrm{Z}\right)$ were manufactured so a mock surgery could be performed. All plaster bone models were acquired from CT images by using a helical CT scanner, and STL models were reconstructed using the same process. By using the final STL models, the preoperative simulation described earlier was performed to identify the bony impingement lesion. The identified bony impingement lesion was reconstructed as an STL model. Registration was performed by combining the fields of view of the Bone Simulator software (Bone Simulator; Orthree) and planning software (Orthomap; Stryker, Kalamazoo, MI, USA). It was visualized by importing the STL model of the identified bony impingement lesion into Orthomap software (Stryker). In this study, only the humerus was subjected to surgical accuracy validation at the coronoid and olecranon fossae, where an osteophyte is likely to form.

\section{Preparing the Arthroscopy Simulator and the Navigation System}

The elbow arthroscopy simulator for this study was similar to that used for validation of the registration accuracy (Fig 3A). ${ }^{14}$ The simulator consisted of the plaster bone models enclosed in rubber skin and spongy subcutaneous tissue (\#1411 arthroscopic elbow, Sawbones; Vashon, WA, USA) and clamps. To simulate elbow arthroscopy in the prone position, with the shoulder at $90^{\circ}$ abduction and the forearm drooping, 
Fig 1. Calculated extension and flexion axis of the elbow. (A) Superimposed 3D models of 3 positions based on the humerus. (B) Calculated rotation axis of the elbow. Extension axis (blue bar) is defined by movement of the ulna between maximum extension and $90^{\circ}$ flexion. Flexion axis (red bar) is defined by movement of the ulna between maximum flexion and $90^{\circ}$ flexion.
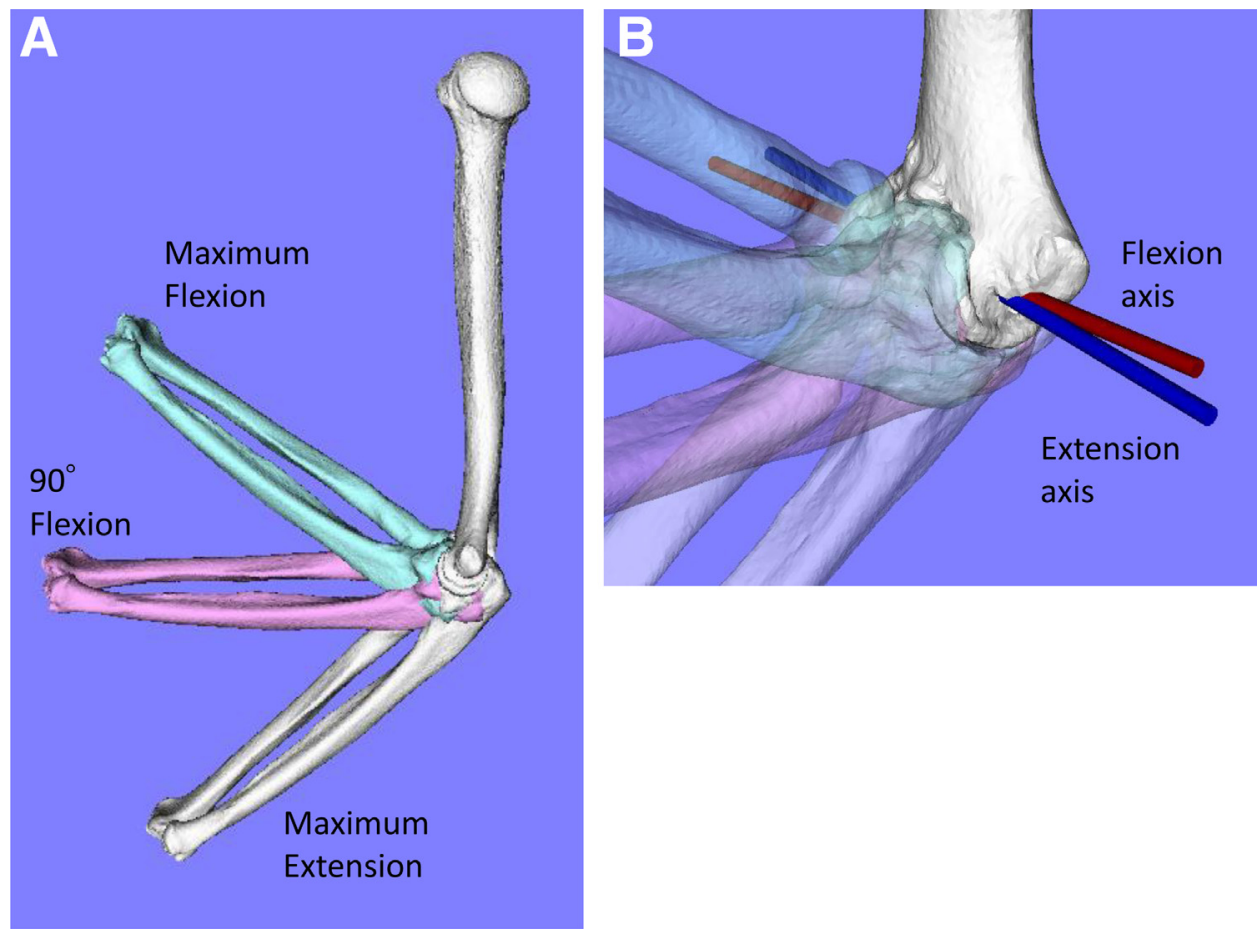

the bone models were fixed at the proximal humerus on a table using a clamp. An arthroscopy tower, including a monitor, camera system (560P High Definition Camera System; Smith \& Nephew, London, UK) and light source (500XL Xenon Light Source; Smith \& Nephew), was set on the proximal side of the humerus. A $2.7 \mathrm{~mm} \times 30^{\circ}$ scope and a 5-portal technique were used for the elbow arthroscopies, operating on the anterior intracapsular areas through the proximal anteromedial and anterolateral portals. Direct posterior, posterolateral and soft-spot portals were used to palpate the posterior intracapsular area.

An optical computer navigation system with a 0.070 $\pm 0.032 \mathrm{~mm}$ optical localizer accuracy ${ }^{18}$ (Stryker Navigation System II Cart; Stryker) was used to perform the navigation procedure. The CT data of the plaster bone model were imported into the 3D navigation and planning software (Orthomap; Stryker) in the DICOM format, and the 3D bone model was created with the same Hounsfield-unit threshold as reconstructed by preoperative simulation software. Once dynamic reference trackers were fixed to the humerus using 23 $\mathrm{mm}$ diameter half pins and clamps of an external fixator system (Hoffman II system; Stryker) through small incisions on the dorsolateral safe zone of the distal humerus. This area is proximal to the lateral epicondyle along the lateral humeral shaft and extends to the level of the lateral epicondyle to the transepicondylar distance where the radial nerve crosses the humerus in the midlateral plane. ${ }^{19}$
Paired-point registration was performed first for the initial alignment, and surface-matching registration based on the iterative closest-point algorithm was secondarily performed for improving the initial alignment by using the navigation pointer. Paired-point registration was performed by digitizing 6 preset anatomic landmarks for the humerus (radial fossa, coronoid fossa, medial and lateral points on the olecranon fossa under arthroscopy, and tips of the medial and lateral epicondyles through small incisions) until a mean deviation of fiducial registration error $<2.0 \mathrm{~mm}$. Surface-matching registration was performed by digitizing 30 points (7 points each on the anterior and posterior intracapsular surfaces, 4 points on the medial epicondyle, 4 points on the lateral epicondyle, and 8 points on the dorsolateral surfaces of the humeral shaft around the insertion of the fixation pin for the humeral tracker) on the bone surface.

\section{Simulated Surgery and Verification for Accuracy of the System}

After the registration procedure, the STL models of the simulated bony impingement lesion were imported into the Orthomap software (Orthomap; Stryker). This allowed us to identify the planned resection lesion on the humerus model on the navigation monitor (Fig 3B). Mock surgery was performed by 3 orthopedic surgeons on 15 plaster bone models enclosed in rubber skin and spongy subcutaneous tissue; they used a $4 \mathrm{~mm}$-diameter blade and control unit 


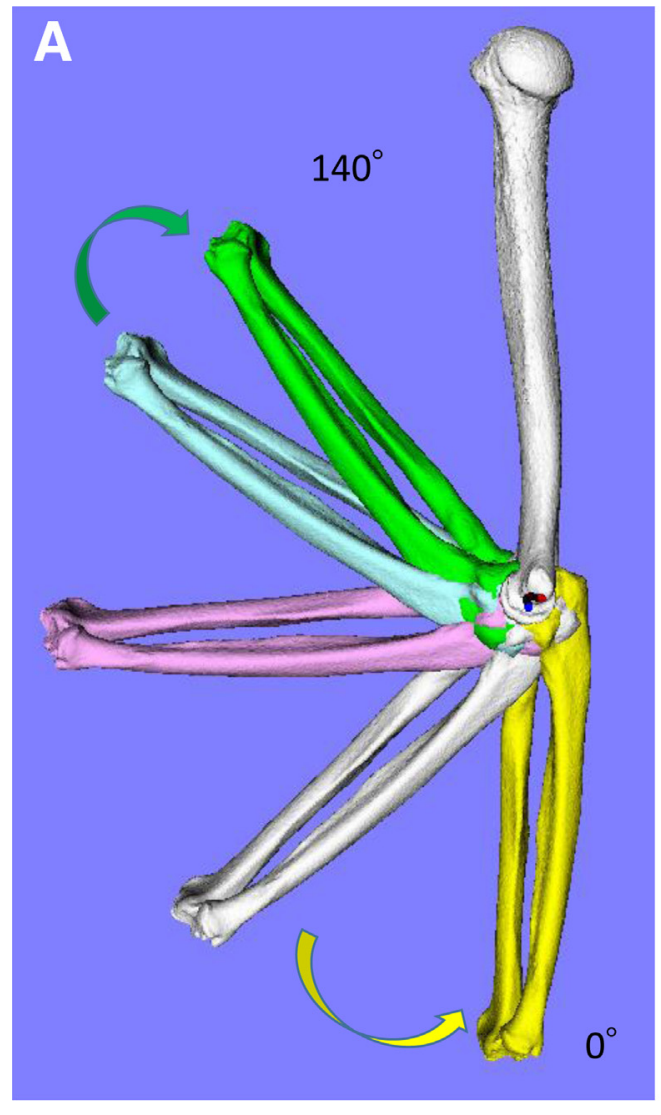

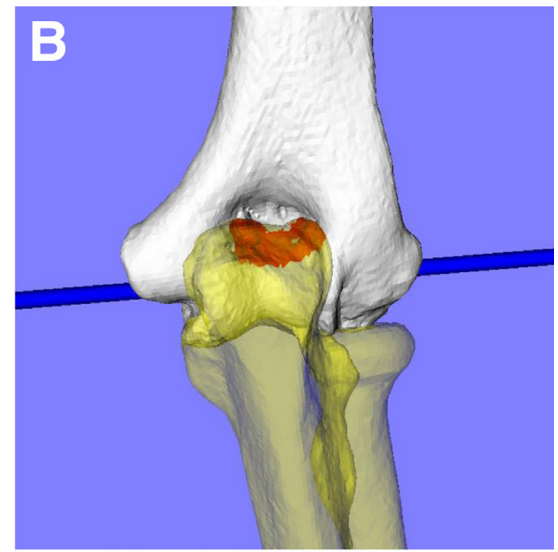

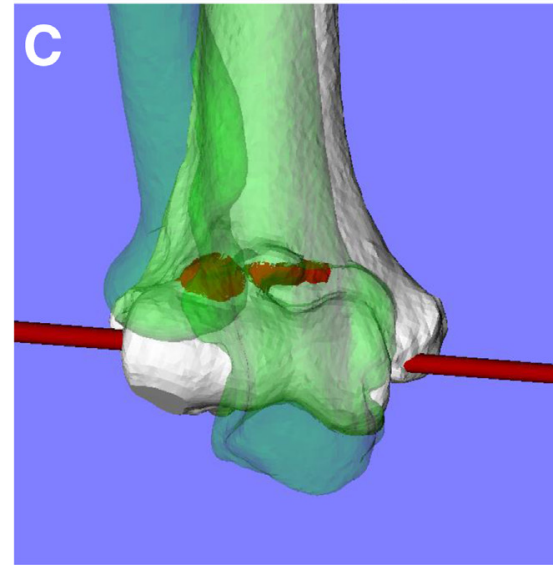

Fig 2. Simulated bony impingement lesion. (A) Simulated bone position at $0^{\circ}$ (yellow bone) and $140^{\circ}$ (green bone) of elbow by rotating the radius and ulna from maximum flexion around the flexion axis and from maximum extension around the extension axis. Simulated bony impingement lesion (red area) in Experiment 2 defined the area where the humerus, radius and ulna overlap. Posterior (B) and anterior $(\mathrm{C})$ views.
(Dyonics Power II; Smith \& Nephew). When the navigation system identified the blade as usual, the distal end of the blade was displayed on the monitor. In actual surgery, the entire spherical blade surface was used to remove osteophytes, so it was necessary to identify the center of the blade tip for the accurate surgery to keep a constant distance between what is displayed on the monitor and the actual surgery site. To make the navigation systems identify the center of the blade tip, the tip of a metal rod $2 \mathrm{~mm}$ shorter than the blade actually used was recognized by the navigation systems in advance and then was replaced with the blade actually used. By this operation, the center of the actual blade was displayed as the tip of the blade in the navigation monitor. Then, displaying a cone with a bottom of $4 \mathrm{~mm}$ diameter and $2 \mathrm{~mm}$ height on the tip of the blade in the navigation monitor helped us to locate the position of $2 \mathrm{~mm}$ from the center of the blade (Fig 3B).

After setting the navigation system, the arthroscopic debridement was performed using computer-aided navigation systems while confirming the simulated resection lesion displayed on the navigation system monitor (Fig 3B). Two types of mock surgery (Experiments 1 and 2) were designed to evaluate the surgical accuracy of the present system. In Experiment 1, the spatial error in excision was measured. Five resection lesions were subjectively decided, including 1 at the radial fossa, 1 at the coronoid fossa and 3 at the olecranon fossa, where osteophytes are likely to form in elbow osteoarthritis, according to a previous report. ${ }^{20} \mathrm{~A}$ $4 \mathrm{~mm}$-diameter sphere with the same diameter as the blade was created at each lesion, whose center was on the bone model surface (Fig 4A). On the navigation monitor, 5 spheres on the bone surface could be confirmed. These 5 resection lesions in the plaster bone model were then resected using computer-aided navigation systems.

In Experiment 2, the resection volume was measured. The bony impingement lesion identified by preoperative simulation was resected. The data for the bony impingement lesion were imported to the navigation system, and the lesion to be removed was displayed on the navigation monitor. Then arthroscopic debridement according to the preoperative simulation was performed using the computer-aided navigation systems for the plaster bone model. When excision was performed, it could be confirmed on the navigation monitor that the tip of the blade entered the resection lesions (Fig 3B). 
A

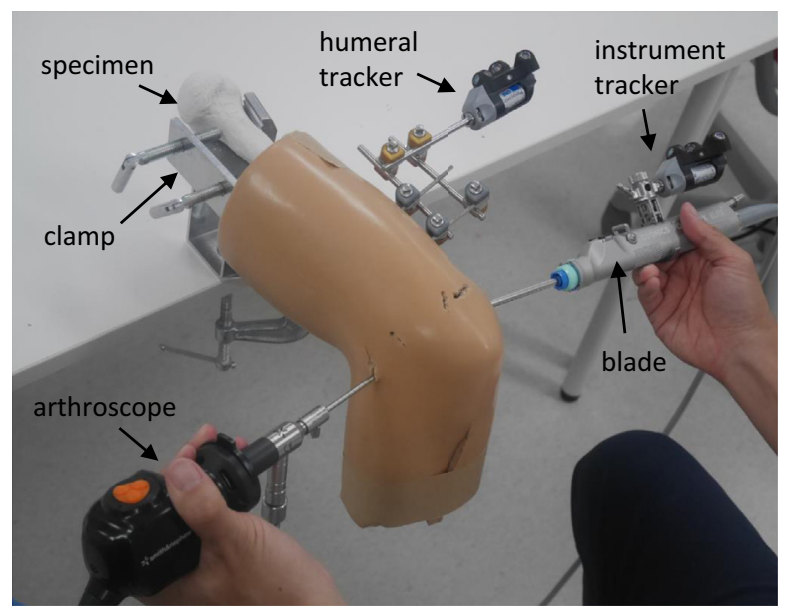

B

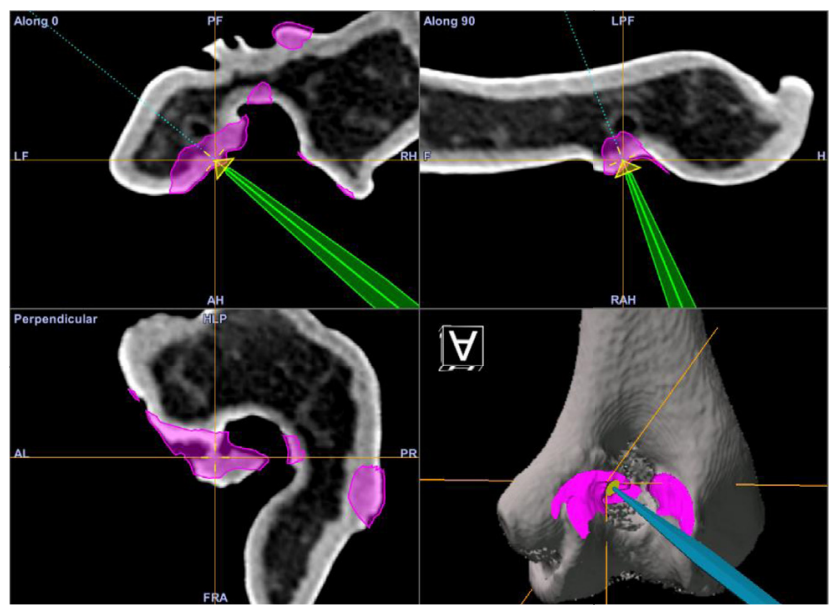

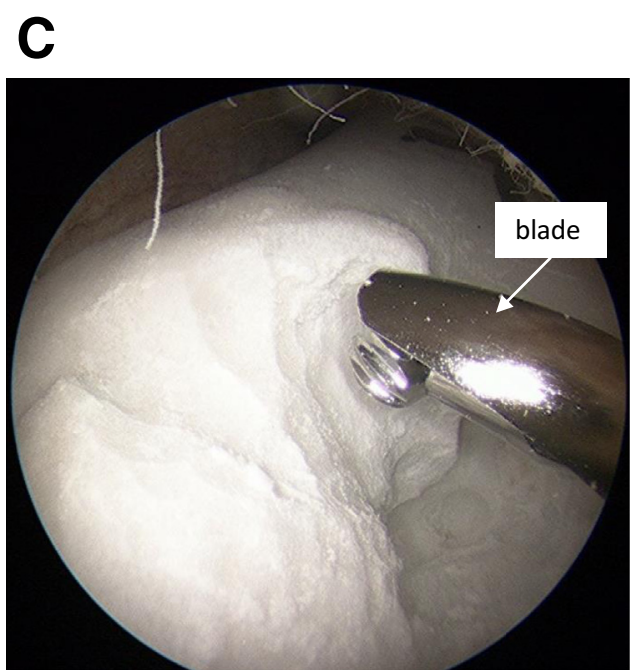

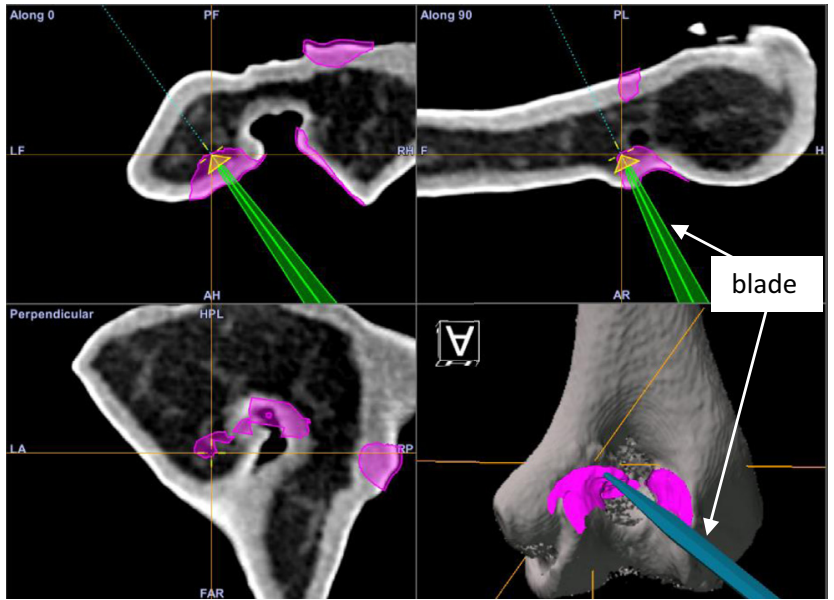

Fig 3. (A) Elbow arthroscopy simulator overview. (B) The navigation system displays the planed resection lesion (pink) on the humerus model. A screw (yellow) with a diameter of $4 \mathrm{~mm}$ and a length of $2 \mathrm{~mm}$ on the tip of the blade. (C) Navigation-assisted arthroscopy combined with preoperative 3D assessment of bony impingement lesions. Left side: Arthroscopic view from the posterolateral portal showing the tip of the calibrated blade. Right side: The navigation system that displays real-time tracking of area for removal (pink) and the tip of the calibrated shaver blade (green or blue) during arthroscopic debridement arthroplasty.

\section{Evaluation of Surgical Accuracy}

After the mock surgery (Experiments 1 and 2), all postoperative plaster models were scanned and CT images of the whole humerus were acquired, again using a helical CT scanner. Postoperative 3D models were reconstructed on the computer and superimposed on the preoperative model by an interactive closest-point algorithm. In Experiment 1, a large number of coordinates of the bone surface of the resected area and the approximate sphere along the bone surface of the resected area were calculated from the acquired coordinates by using the coordinate system of the Bone Simulator software (Fig 4B). The distance between the center of the approximate sphere and that of the preoperative image was calculated (Fig 4C). In Experiment 2, the 3D resection volume was calculated by subtracting the postoperative from the preoperative bone models using a Boolean arithmetic operation and Magics software 23.0 (Materialise, Leuven, Belgium). The simulated and actual resection volumes were compared to assess how much overresection occurred and how much the planned area was resected during the mock surgery. The surgical accuracy was evaluated using the mean value of the center-to-center errors and the difference of resection volumes between the simulation and the mock surgery in 45 trials. The differences in surgical accuracy among the 3 surgeons were also evaluated using the mean of 15 trials. The same surgeon performed 30 trials, and the difference in surgical accuracy between the first and second halves was also evaluated using the mean of 15 trials. 


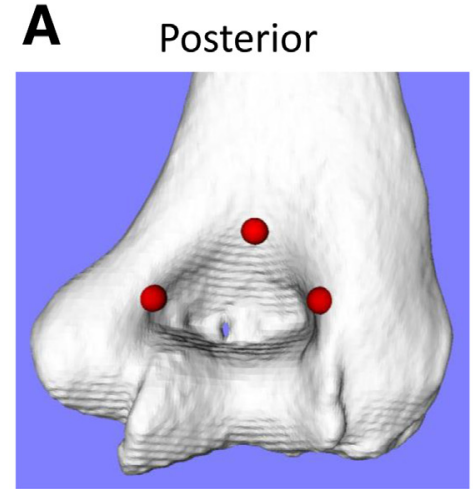

Anterior

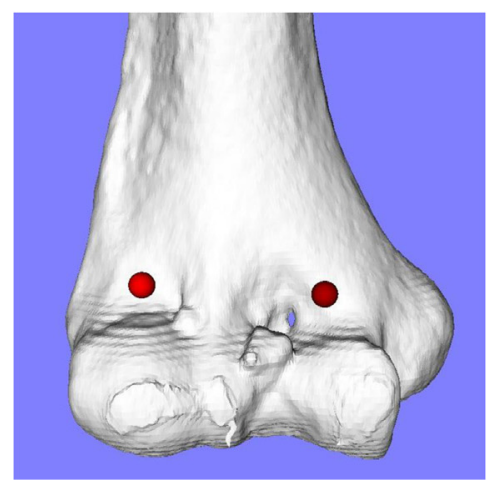

B

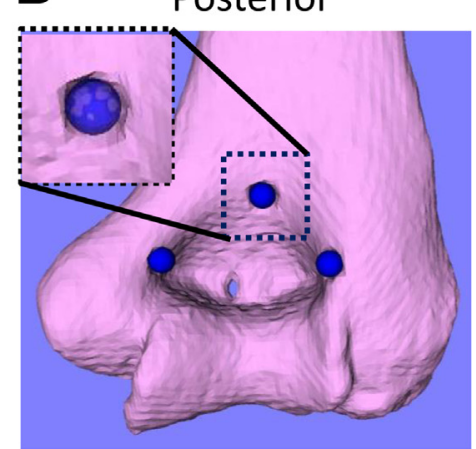

Anterior

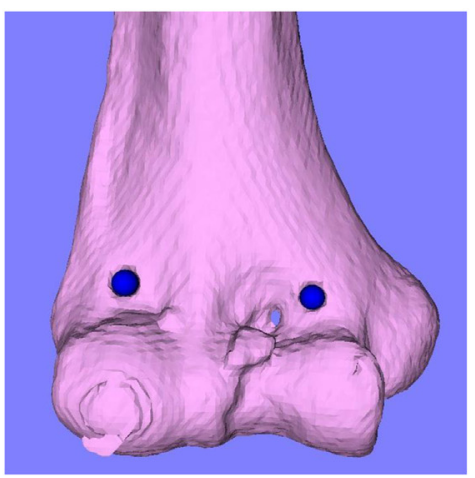

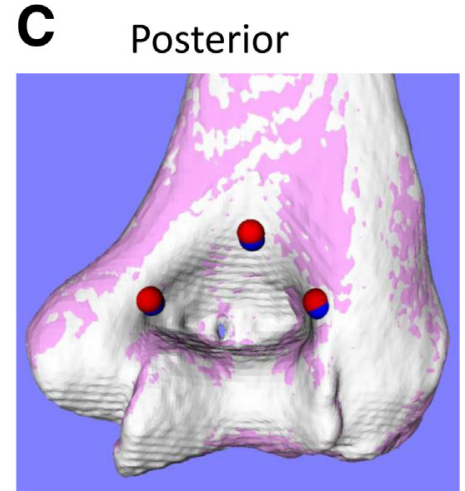

Anterior

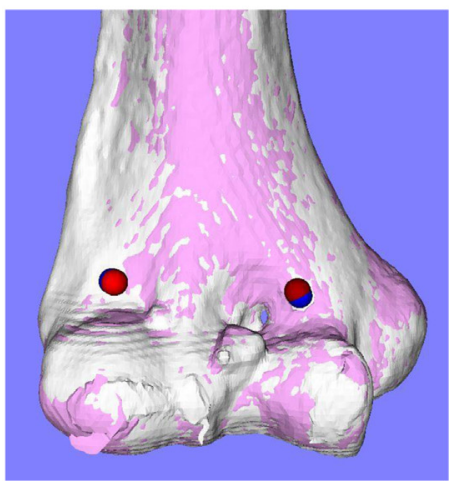

Fig 4. Evaluation of the center-to-center error between the simulated area for removal and the actual resected area in Experiment 1. (A) The sphere of the simulated area for removal: 1 at the radial fossa, 1 at the coronoid fossa and 3 at he olecranon fossa. (B) The approximate sphere of the actual resected area. (C) Superposition of 2 models.

\section{Statistical Analysis}

All statistical analyses were performed using the JMP Pro 14 software (SAS, Cary, NC, USA). Data were assessed for normal distribution using the Shapiro-Wilk test. We used parametric and nonparametric statistical analyses for normally and non-normally distributed data, respectively.

Differences in the center-to-center error between anterior and posterior joint surgery accuracy were evaluated using the Wilcoxon test. Differences in resection volume between anterior and posterior joint surgery accuracy were evaluated using a paired $t$ test. Differences in over-resection volume among the 3 surgeons and by the same surgeon were evaluated using the intrarater reliability and inter-rater reliability. A $P$ value $<0.05$ was considered statistically significant.

Power analysis was performed with G-power 3.1 (University of Kiel, Kiel, Germany). For sample size calculation, $\alpha$, power and effect size were set. No specific index for power analysis was obtained from previous studies, so power analysis was conducted assuming an effect size of 0.8 , which is generally judged to have a large effect size in paired $t$ tests. The analysis indicated that 15 trials were required to achieve a statistical significance level of 0.05 , a power of $80 \%$ and an effect size of 0.8 for paired $t$ tests.

\section{Results}

In Experiment 1, the mean center-to-center error between the simulated sphere and the actual resection was $1.13 \mathrm{~mm}$ (median, 1.04; IQR, 0.78 to 1.42 ). Mean center-to-center errors in the posterior 3 and anterior 2 areas were 1.03 (median, 1.00; IQR, 0.74 to 1.31 ) and 1.27 (median, 1.19; IQR, 0.89-1.31) mm, respectively (Fig 5). There was a significant difference in the centerto center error between the posterior and anterior areas $(P=0.0008)$.

In Experiment 2, the extent of over-resection was evaluated. Mock surgery resulted in a mean of $8 \pm 15 \%$ (median, 7; IQR, -5 to 18 ) excessive bone resection over the preoperative simulations. In the anterior joint, a mean of $0 \pm 18 \%$ (median, 2 ; IQR, -13 to 12 ) more bone was resected than in the simulation. In the posterior joint, a mean of $16 \pm 19 \%$ (median, 15; IQR, 1 to 33) more bone was resected than the simulation (Fig 6). There was a significant difference in the over-resection volume between the anterior and posterior areas $(P=$ $0.0003)$. Intra-rater reliability was intraclass correlation $(\mathrm{ICC})_{2,1} 0.81$ and inter-rater reliability was ICC $_{1,1} 0.87$ (Table 2).

In addition, the extent of resection of the planned area was evaluated. A mean of $85 \%$ (median, 84; IQR, 78 to 91 ) was resected in the preoperative simulated 

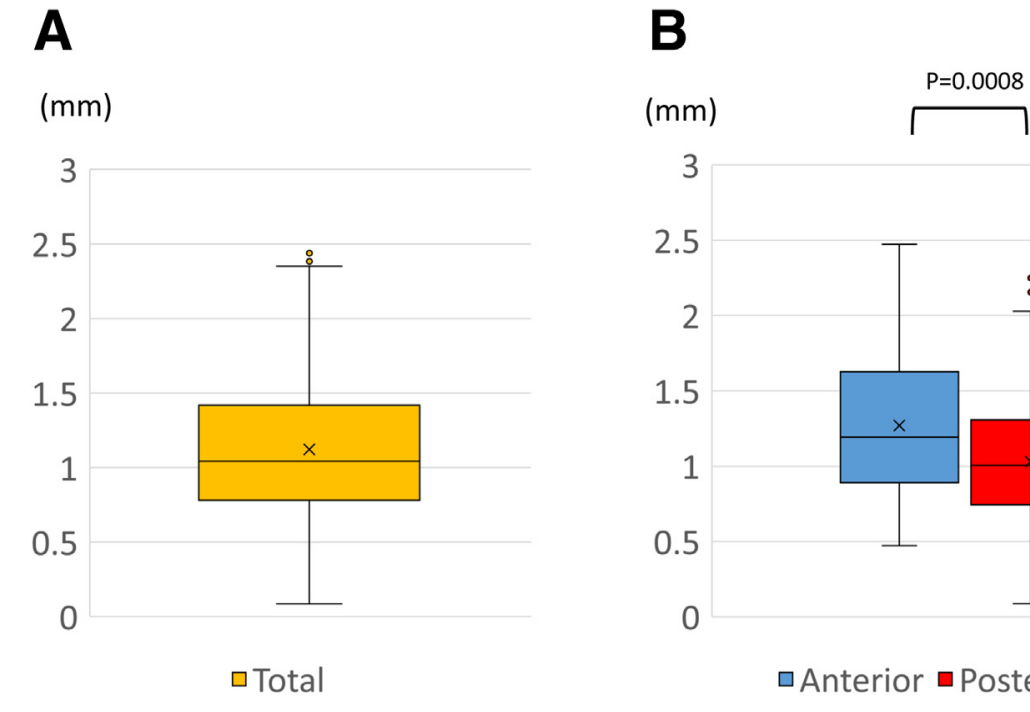

Fig 5. Results of the center-tocenter error ( $\mathrm{mm})$ between simulated area to remove and actual resected area in Experiment 1. (A) The surgical accuracy of the total area, including the anterior and posterior joints. (B) The surgical accuracy of each site. Open circles represent statistical outliers.

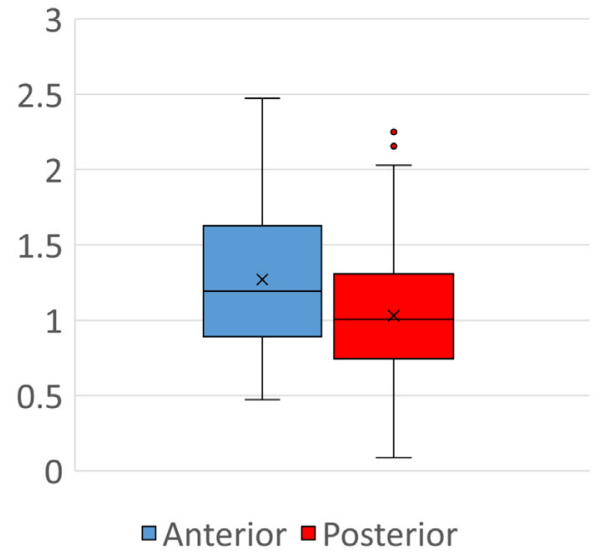

Wilcoxon test

site using mock surgery. In the anterior joint, a mean of $90 \%$ (median, 90; IQR, 85 to 95) was resected at the preoperative simulated area. In the posterior joint, a mean of $82 \%$ (median, 81 ; IQR, 74 to 90 ) was resected at the preoperative simulated area.

\section{Discussion}

In this study, we found that the bony impingement lesion in elbow osteoarthritis can be removed accurately, as planned during arthroscopy, by using a computer-aided navigation system. Arthroscopic debridement of the elbow is a valuable surgical treatment for patients with elbow osteoarthritis to improve symptoms, such as loss of extension and flexion and pain at the range of motion endpoints. ${ }^{1,7-10}$ On the other hand, some problems exist in identifying the impinging osteophyte accurately during surgery, ${ }^{21}$ and surgical results often depend on the skill of the operator. To solve these problems, we devised a method that combines preoperative dynamic simulation using computer-rendered 3D models to identify the bony impingement lesion and navigation systems and confirm the resection lesion during surgery.

The use of computer-aided surgery in orthopedics has been applied to various procedures, such as implantation of components and arthroscopic surgeries of the hip, shoulder, knee, and ankle, ${ }^{22,23}$ with surgical accuracy of the entire computer-assisted application

\section{A}

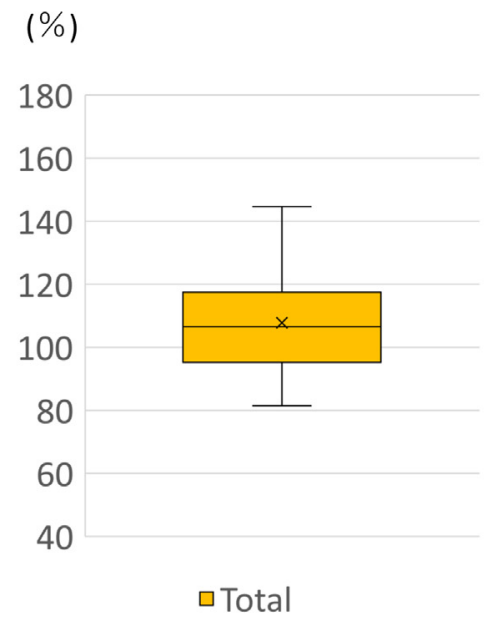

B

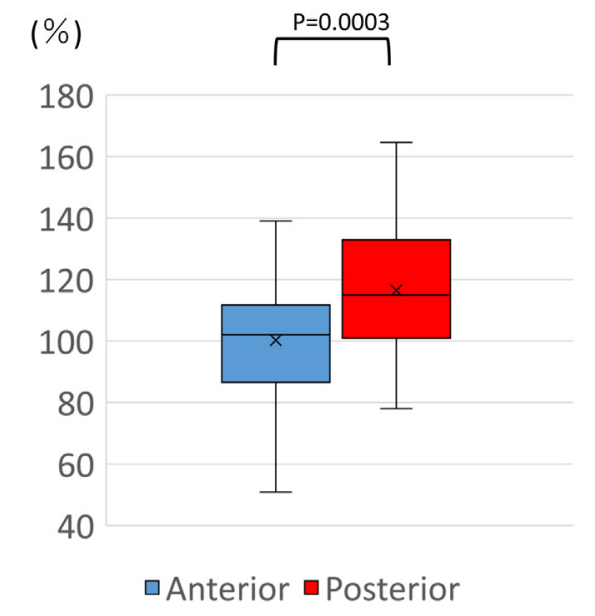

Fig 6. Results of actual resection volume $(\%)$ for plan in Experiment 2. (A) The surgical accuracy of the total area, including the anterior and posterior joints. (B) The surgical accuracy of each site. 


\section{A Posterior surgery}

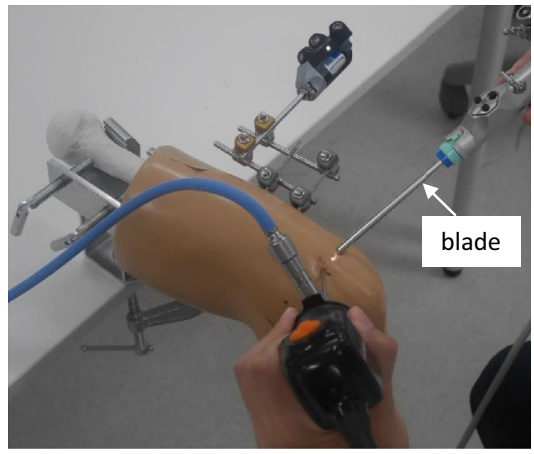

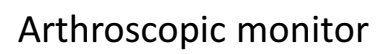

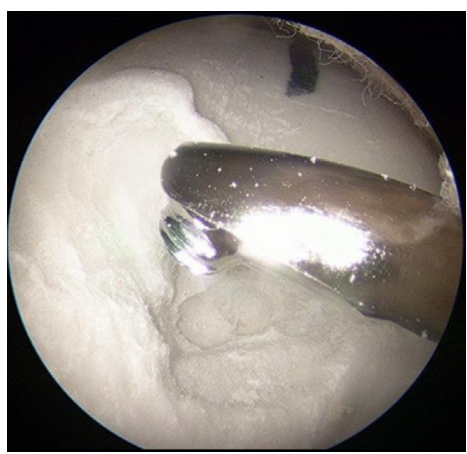

Navigation monitor

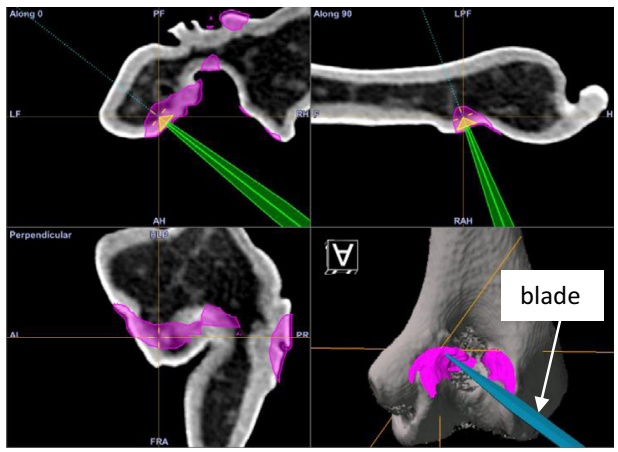

\section{B Anterior surgery}

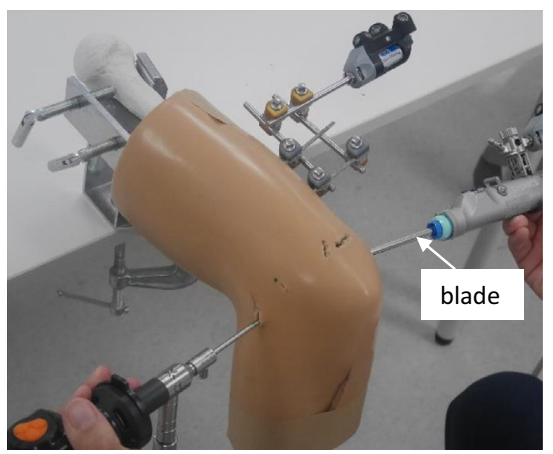

\section{Arthroscopic monitor}

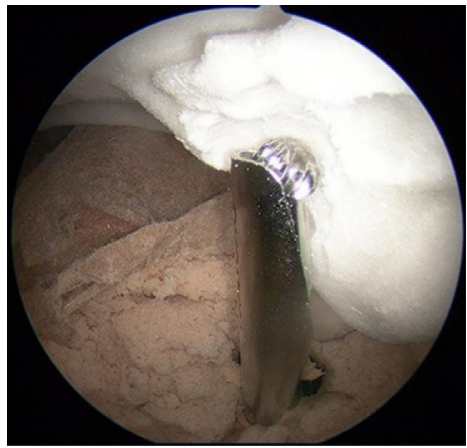

Navigation monitor

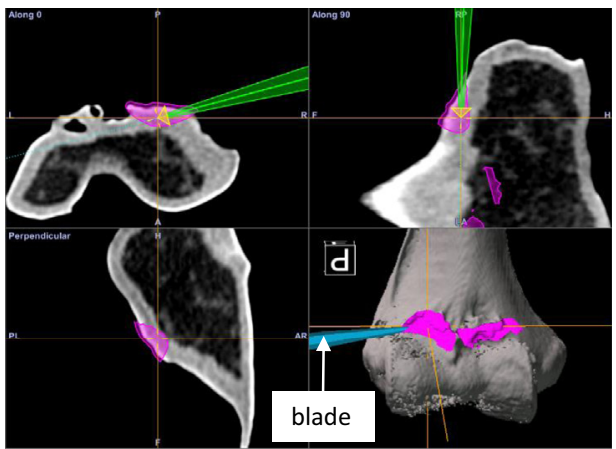

Fig 7. Difference between posterior (A) and anterior (B) surgery. The blade is inserted from the same direction, but the display direction of the blade on the navigation monitor is different.

ranging from 1.2 to $2.0 \mathrm{~mm} .{ }^{24-26}$ Navigation assistance for elbow surgery has been reported in implantation of components for total elbow arthroplasty. ${ }^{26}$ In this study, the translational and rotational errors of humeral component implantation were $1.2 \pm 0.3 \mathrm{~mm}$ and $1.3 \pm$ 0.3 degrees in the cadaveric study. Our system also had high accuracy comparable to the accuracy in other joints, with a $1.13 \mathrm{~mm}$ (median, 1.04; IQR, 0.78 to 1.42) error in mock surgery, which is very close to actual surgery.

Open arthroplasty assisted by a navigation system for elbow osteoarthritis reportedly led to significant improvement in clinical symptoms. ${ }^{27}$ It was reported that the ratio of resection volume relative to the preoperative plan was $95.9 \% \pm 2.5 \%$ in the coronoid fossa and $95.8 \% \pm 3.7 \%$ in the olecranon fossa. However, that study differed from our study in that it was open surgery, and the setting of the planned resection area and the evaluation of the resection volume were performed manually in 2 dimensions using the sagittal plane of CT. The average amount of over-resection was $17 \%$ for removal of a femoral acetabular impingement lesion with the use of computer-aided navigation systems during arthroscopic hip surgery. ${ }^{22}$ An error of resection volume was suggested to occur because the navigation monitor displays the distal tip of the drill,

Table 1. Surgical Accuracies by Each Surgeon and all Surgeons in Experiment 2

\begin{tabular}{llcccc}
\hline & & \multicolumn{2}{c}{ Over-resection volume, $\%$} & Intrarater reliability \\
\cline { 3 - 5 } \cline { 3 - 6 } & $\mathrm{n}$ & Mean & Standard deviation & Median & Interquartile range $^{\text {ICC }_{2,1}}$ \\
\hline Surgeon 1 & 15 & 104 & 12 & 101 & $93-117$ \\
Surgeon 2 & 15 & 112 & 17 & 112 & $95-132$ \\
Surgeon 3 & 15 & 108 & 15 & 106 & $95-122$ \\
Total & 45 & 108 & 15 & 107 & $95-118$ \\
\hline
\end{tabular}


Table 2. Surgical Accuracies by the Same Surgeon (surgeon 1) in Experiment 2

\begin{tabular}{lccccc}
\hline & & \multicolumn{2}{c}{ Over-resection volume, mm } & Inter-rater reliability \\
\cline { 2 - 5 } \cline { 3 - 6 } & $\mathrm{n}$ & Mean & Standard deviation & Median & Interquartile range $^{\text {ICC }_{1,1}}$ \\
\hline First half & 15 & 119 & 36 & 130 & $84-151$ \\
Second half & 15 & 104 & 12 & 101 & $93-117$ \\
Total & 30 & 112 & 28 & 104 & $92-131$ \\
\hline
\end{tabular}

although the bone actually was excised on the entire surface of the drill. In our study, the average amount of over-resection was only $8 \%$, which was comparable to or superior to $17 \%$ for the removal of a femoral acetabular impingement lesion. The reason for the improved average amount of over-resection was considered to be visualization of the center of the blade on the navigation monitor. With this operation, the distance between the drill tip visualized on the monitor and the actual cutting surface could be kept constant. Also, in our study, $85 \%$ of the preoperative simulated area was resected. However, in our experiments, the surgical accuracy of the anterior joint was significantly lower than that for the posterior joint. This is probably because the hand of the drill operator was oriented in the same direction as the blade when viewed on the arthroscopic monitor during the posterior surgery, but the hand of the drill operator was oriented in the reverse direction from the blade when viewed on the arthroscopic monitor during the anterior surgery (Fig 7). However, both intrarater reliability and inter-rater reliability were high (Table 1) (Table 2); accurate surgery would be expected independent of the skill of the operator.

In summary, this preliminary validation study of surgery using CT-based navigation systems for arthroscopic debridement of the elbow provided accuracy comparable to that in the other joints. Our method, which combines a computer-aided navigation system with visualization of osteophytes by preoperative dynamic 3D simulation, provides real-time tracking of both surgical instruments and bony impingement lesions and also solves the technical difficulties of arthroscopic surgery of the elbow joint.

\section{Limitations}

This study has several limitations. First, we performed experiments using plaster bone models created from CT data of actual patients with elbow osteoarthritis and mimicked soft tissues to approximate the conditions in actual surgery. However, we did not include cartilage, capsules or synovium in the model, and that could have affected the accuracy of registration and surgery. Second, we examined only the humerus in this study, and it was unclear whether the same accuracy was obtained for the radius and ulna. However, we considered that the surgical accuracy of the humerus alone was sufficient for clinical use. Third, our method had a problem with radiation exposure, which required $\mathrm{CT}$ imaging of 3 positions in actual clinical practice. However, the total radiation dose for 3 scans in our low-dose protocol was about 1/10 smaller than that for the 1 CT scan that was normally used, so we did not consider it to be an overdose.

\section{Conclusions}

Surgery using computer-aided navigation systems for arthroscopic debridement of the elbow provided accuracy comparable to that in other joints.

\section{Acknowledgments}

The authors thank Hisashi Tanaka (radiologist) for collaboration on this work. We also thank Yasuhiro Yanagawa (radiology technologist) for assistance with the experiments. We are grateful to Ryouji Nakao (computer programmer) for contributing to the study. We acknowledge the contribution of Toru Iwahashi and Arisa Kazui to the study conception and design.

\section{References}

1. Adams JE, Wolff LH 3rd, Merten SM, Steinmann SP. Osteoarthritis of the elbow: Results of arthroscopic osteophyte resection and capsulectomy. J Shoulder Elbow Surg 2008;17:126-131.

2. Antuna SA, Morrey BF, Adams RA, O'Driscoll SW. Ulnohumeral arthroplasty for primary degenerative arthritis of the elbow: Long-term outcome and complications. J Bone Joint Surg Am 2002;84:2168-2173.

3. Sarris I, Riano FA, Goebel F, Goitz RJ, Sotereanos DG. Ulnohumeral arthroplasty: Results in primary degenerative arthritis of the elbow. Clin Orthop Relat Res 2004;420: 190- 193.

4. Yamamoto M, Murakami Y, Iwatsuki K, Kurimoto S, Hirata H. Feasibility of four-dimensional preoperative simulation for elbow debridement arthroplasty. BMC Musculoskelet Disord 2016;17:144.

5. Suvarna SK, Stanley D. The histologic changes of the olecranon fossa membrane in primary osteoarthritis of the elbow. J Shoulder Elbow Surg 2004;13:555-557.

6. Tsuge K, Mizuseki T. Debridement arthroplasty for advanced primary osteoarthritis of the elbow: Results of a new technique used for 29 elbows. J Bone Joint Surg Br 1994;76:641-646.

7. O'Driscoll SW, Morrey BF. Arthroscopy of the elbow: Diagnostic and therapeutic benefits and hazards. J Bone Joint Surg Am 1992;74:84-94. 
8. Galle SE, Beck JD, Burchette RJ, Harness NG. Outcomes of elbow arthroscopic osteocapsular arthroplasty. J Hand Surg Am 2016;41:184-191.

9. Kelly EW, Bryce R, Coghlan J, Bell S. Arthroscopic debridement without radial head excision of the osteoarthritic elbow. Arthroscopy 2007;23:151-156.

10. Krishnan SG, Harkins DC, Pennington SD, Harrison DK, Burkhead WZ. Arthroscopic ulnohumeral arthroplasty for degenerative arthritis of the elbow in patients under fifty years of age. J Shoulder Elbow Surg 2007;16: 443-448.

11. Lim TK, Koh KH, Lee HI, Shim JW, Park MJ. Arthroscopic debridement for primary osteoarthritis of the elbow: Analysis of preoperative factors affecting outcome. J Shoulder Elbow Surg 2014;23:1381-1387.

12. Cheung EV, Adams R, Morrey BF. Primary osteoarthritis of the elbow: Current treatment options. J Am Acad Orthop Surg 2008;16:77-87.

13. Miyake J, Shimada K, Oka K, et al. Arthroscopic debridement in the treatment of patients with osteoarthritis of the elbow, based on computer simulation. Bone Joint J 2014;96-B:237-241.

14. Shigi A, Oka K, Tanaka H, et al. Validation of the registration accuracy of navigation-assisted arthroscopic debridement for elbow osteoarthritis. J Shoulder Elbow Surg 2019;28:2400-2408.

15. Oka K, Murase T, Moritomo H, Goto A, Sugamoto K, Yoshikawa H. Accuracy analysis of three-dimensional bone surface models of the forearm constructed from multidetector computed tomography data. Int J Med Robot 2009;5:452-457.

16. Omori S, Murase T, Kataoka T, et al. Three-dimensional corrective osteotomy using a patient-specific osteotomy guide and bone plate based on a computer simulation system: Accuracy analysis in a cadaver study. Int $J$ Med Robot 2014;10:196-202.

17. Oura K, Oka K, Kawanishi Y, Sugamoto K, Yoshikawa H, Murase T. Volar morphology of the distal radius in axial planes: A quantitative analysis. J Orthop Res 2015;33: 496-503.
18. Elfring R, de la Fuente M, Radermacher K. Assessment of optical localizer accuracy for computer-aided surgery systems. Comput Aided Surg 2010;15:1-12.

19. Kamineni S, Ankem H, Patten DK. Anatomic relationship of the radial nerve to the elbow joint: Clinical implications of safe pin placement. Clin Anat 2009;22:684-688.

20. Miyake J, Shimada K, Moritomo H, Kataoka T, Murase T, Sugamoto K. Kinematic changes in elbow osteoarthritis: In vivo and 3-dimensional analysis using computed tomographic data. J Hand Surg Am 2013;38: 957-964.

21. Nishiwaki M, Willing R, Johnson JA, King GJ, Athwal GS. Identifying the location and volume of bony impingement in elbow osteoarthritis by 3-dimensional computational modeling. J Hand Surg Am 2013;38:1370-1376.

22. Audenaert E, Smet B, Pattyn C, Khanduja V. Imageless versus image-based registration in navigated arthroscopy of the hip: A cadaver-based assessment. J Bone Joint Surg Br 2012;94:624-629.

23. Verborgt O, De Smedt T, Vanhees M, Clockaerts S, Parizel PM, Van Glabbeek F. Accuracy of placement of the glenoid component in reversed shoulder arthroplasty with and without navigation. J Shoulder Elbow Surg 2011;20:21-26.

24. Iwana D, Nakamura N, Miki H, Kitada M, Hananouchi T, Sugano N. Accuracy of angle and position of the cup using computed tomography-based navigation systems in total hip arthroplasty. Comput Aided Sur 2013;18: 187-194.

25. Amiot LP, Poulin F. Computed tomography-based navigation for hip, knee, and spine surgery. Clin Orthop Relat Res 2004;421:77-86.

26. McDonald CP, Johnson JA, Peters TM, King GJ. Imagebased navigation improves the positioning of the humeral component in total elbow arthroplasty. J Shoulder Elbow Surg 2010;19:533-543.

27. Ikeda M, Kobayashi Y, Saito I, Ishii T, Shimizu A, Oka Y. Application of a navigation system for contouring anatomical plasty of the distal end of the humerus. Comput Aided Surg 2012;17:179-186. 